\title{
Estimation of a local 1D or 2D thermal conductivity field with infrared images processing and volume averaging method
}

\author{
By Marielle VARENNE, Jean-Christophe BATSALE, Claire GOBBE
}

L.E.P.T.-ENSAM, UMR 8508 CNRS, Esplanade des Arts and Metiers - 33405 Talence Cedex FRANCE - Phone : (33) 556845429 -Fax : (33) 556845436

E-mail : batsale@lept-ensam.u-bordeaux.fr

\begin{abstract}
The estimation of local thermophysical properties can be of paramount importance in order to study heterogeneous media. The volume averaging method is used to implement an estimation method of a local thermal conductivity field of a $1 \mathrm{D}$ or $2 \mathrm{D}$ heterogeneous medium. The method is tested with experimental transient temperature fields, obtained with a calibrated sample with an infrared camera. A large number of images are processed at transient state. The same intrinsic stationary field is estimated from each image. By repeated estimations, this method reduces the measurement noise influence.
\end{abstract}

\section{Introduction}

The aerospace and electronic industries use a lot of composite materials. If the macroscopic thermophysical properties are often sufficient for designers, it may be of importance to characterize the local behavior of such materials. The most commonly used methods to estimate local properties consist in doing local scale experiments. These methods, such as laser photoreflectance (Lepoutre et al 1996), need to handle very short characteristic times. The method proposed here tries to implement a macroscopic experiment in order to estimate local properties. The basic principle is to consider that, for longer times, the local transfer is steady, even if the transfer at a larger scale is transient. Therefore, the experimental device, based on infrared thermography, is simple. Nonetheless, the obtained images are quite noisy and complex, and must be processed using physical models. The volume averaging methods (Quintard and Whitaker 1993) is here used to process infrared images. This method is ordinary used to compute effective macroscopic properties (Nozad et al 1985). Here, transient temperature fields are processed in order to estimate local thermophysical properties.

In this paper, a brief description of the method of volume averaging is made. Then, we describe its application to the estimation of the local thermal conductivities of a 1D and 2D heterogeneous medium.

\section{Presentation of the volume averaging method}

The volume averaging method (Marle 1967 and Quintard and Whitaker 1993) allows us to describe the transfers in a heterogeneous medium through a macroscopic model associated to an equivalent continuous medium. The basic idea of the volume averaging theory is that a temperature field $T$ is the sum of a mean component, or macroscopic field $\langle T\rangle$, and of a local component, $\tilde{T}$, which describes the spatial deviation around the mean value of the temperature:

$$
T=\langle T\rangle+\tilde{T} .
$$


Here, the macroscopic temperature $\langle T\rangle$ is an average temperature upon a surface of the same size as the unit cell of the periodic heterogeneous medium. In the general case, $\langle T\rangle$ is defined by a convolution product such as:

$$
\langle T\rangle=m \otimes T \text {. }
$$

Where $m$ is a gate function of characteristic length $l$. The previously described averaging method also corresponds to a filtering of the signal. It attenuates the fluctuations of spatial frequency greater than the characteristic frequency $1 / l$, if $l$ is the length of the unit cell.

Carbonell and Whitaker (1984) propose that local variables (spatial deviations $\tilde{T}$ ) be linked with macroscopic variables (macroscopic temperature gradient field $\nabla\langle T\rangle$ ) by:

$$
\tilde{T}=\mathbf{b} . \nabla\langle T\rangle \text {. }
$$

In a general case, $\mathbf{b}$ is a vector. The components of the closure vector $\mathbf{b}$ are local coefficients and depend on both the geometry and the thermophysical properties of the medium. When Eq. (3) is introduced into local equations, it yields a closure problem, which links b-field and the thermal conductivity $\lambda$-field (Quintard and Whitaker 1993):

$$
\nabla \cdot(\lambda \nabla \mathbf{b})=-\nabla \cdot(\lambda \mathbf{I})
$$

Eq. (4) is stationary. This is due to the fact that the characteristic times related to transfer at a local scale are much smaller than the times related to transfer between the excitation and the location of the measurement.

\section{Experimental estimation of a local thermal conductivity cartography}

The experimental method consists in applying a transient heat flux on one edge of a heterogeneous sample (see Fig. 1). For each time step of the experiment, an infrared camera records the temperature field at the surface of the sample.

\subsection{Estimation of the solution of the closure problem}

Each infrared image gives a 1D or 2D-temperature field $T$. The macroscopic temperature field $\langle T\rangle$ is deduced from each temperature field by use of Eq. (2). The spatial deviation field, $\widetilde{T}$, is deduced from fields $T$ and $\langle T\rangle$, by use of Eq. (1). The macroscopic temperature gradient field, $\nabla\langle T\rangle$, is obtained by differentiating $\langle T\rangle$ with respect to the main heat flux directions. In 2D case, , it has two components: $\nabla_{x}\langle T(x, y, t)\rangle$ and $\nabla_{y}\langle T(x, y, t)\rangle$.

In the case of a periodical 1D medium, $\mathbf{b}$ becomes a scalar field $b(x)$. In the 2D case, $\mathbf{b}$ becomes a vector field whose components depend on both space variables $x$ and $y$ :

$$
\mathbf{b}(x, y)=\left[\begin{array}{ll}
b^{x}(x, y) & b^{y}(x, y)
\end{array}\right]
$$

In our experiments, the sample is heated on one of its edges (Fig. 1). Consequently, the macroscopic temperature gradient is negligible in the direction perpendicular to the main heat flux. The unit cell is symmetric and the components $b^{x}$ and $b^{y}$ of $\mathbf{b}$-field are of the same order of magnitude. Those last two remarks yield a simplification in the expression of the closure problem. If the main heat flux is along $O x$ direction, respectively $O y$ direction, then the following relations approximate the closure problem (Eq. 3):

$$
\widetilde{T}(x, y, t) \approx b^{x}(x, y) \nabla_{x}\langle T(x, y, t)\rangle,
$$


respectively:

$$
\widetilde{T}(x, y, t) \approx b^{y}(x, y) \nabla_{y}\langle T(x, y, t)\rangle .
$$

In theory, one thermographic image is enough to estimate the $b^{x}$ or $b^{y}$-field. But all the images need to be processed, in order to have an average value of the $\mathbf{b}$-field, so as to decrease the noise influence. In 2D case, the knowledge of the b-field requires two experimental estimations, one for the $b^{x}$-field, and one for the $b^{y}$-field. The conductivity field is then deduced from the estimation of $\mathbf{b}$-field (Eq. 4).

\subsection{Estimation of local thermal conductivities}

In 1D heterogenous medium, Eq. (4) is written :

$$
\frac{d}{d x}\left(\lambda(x) \frac{d b(x)}{d x}\right)=-\frac{d \lambda(x)}{d x}
$$

and the expression of $\lambda$-field is then obtained directly:

$$
\lambda(x)=\lambda_{1} \lambda_{2} /\left(e_{2} \lambda_{1}+e_{1} \lambda_{2}\right) /\left(1+\frac{d b(x)}{d x}\right)
$$

In a 2D medium, Eq. (4) is more complex and is written:

$$
\left\{\begin{array}{l}
\frac{\partial}{\partial x}\left(\lambda(x, y) \frac{\partial b^{x}(x, y)}{\partial x}\right)+\frac{\partial}{\partial y}\left(\lambda(x, y) \frac{\partial b^{x}(x, y)}{\partial y}\right)=-\frac{\partial \lambda(x, y)}{\partial x} \\
\frac{\partial}{\partial x}\left(\lambda(x, y) \frac{\partial b^{y}(x, y)}{\partial x}\right)+\frac{\partial}{\partial y}\left(\lambda(x, y) \frac{\partial b^{y}(x, y)}{\partial y}\right)=-\frac{\partial \lambda(x, y)}{\partial y}
\end{array} .\right.
$$

The estimation of $\lambda$-field from the $\mathbf{b}$-field is an ill posed problem which is very sensitive to the measurement noise. In 1D case, the main difficulty is to handle the derivation of $b$ field. In order to study analytically, in 2D case, the measurement noise influence on the inversion, an approximated asymptotic solution of Eq. (10) is searched for. The $\mathbf{b}$ and $\lambda$ fields are decomposed according to a perturbation method (Aziz 1988):

$$
\begin{aligned}
& \mathbf{b}(x, y)=\mathbf{b}_{0}(x, y)+\eta \mathbf{b}_{1}(x, y), \\
& \lambda(x, y)=\lambda_{0}(x, y)+\eta \lambda_{1}(x, y)+O\left(\eta^{2}\right),
\end{aligned}
$$

where $\eta$ is the perturbation parameter. $\lambda_{0}$ stands for the equivalent, or macroscopic, thermal conductivity of the heterogeneous medium. This latter conductivity has to be deduced from a macroscopic measurement of the thermal diffusivity (Varenne et al 2000), when the global heat capacity is previously estimated.

Equations (5), (11) and (12) are used to rewrite system (10), which yields several systems, related to each order of parameter $\eta$.

The order 0 solution is $b_{0}^{x}(x, y)=b_{0}^{y}(x, y)=0$, because of the periodicity conditions on $\mathbf{b}$ field (Quintard and Whitaker 1993). The order 1 system is:

$$
\left\{\begin{array}{l}
\lambda_{0}\left(\frac{\partial^{2} b_{1}^{x}(x, y)}{\partial x^{2}}+\frac{\partial^{2} b_{1}^{x}(x, y)}{\partial y^{2}}\right)=-\frac{\partial \lambda_{1}(x, y)}{\partial x} \\
\lambda_{0}\left(\frac{\partial^{2} b_{1}^{y}(x, y)}{\partial x^{2}}+\frac{\partial^{2} b_{1}^{y}(x, y)}{\partial y^{2}}\right)=-\frac{\partial \lambda_{1}(x, y)}{\partial y}
\end{array} .\right.
$$

A double integral transform, based on a double spatial Fourier transform, is applied to each equation of system (13). It yields a system, which links the first order of the decomposition of the $\lambda_{1}$ and $\mathbf{b}_{1}$-field in Fourier space: 


$$
\left\{\begin{array}{l}
\Lambda_{1}\left(\alpha_{n}, \beta_{m}\right)=\frac{\lambda_{0}\left(\alpha_{n}^{2}+\beta_{m}^{2}\right)}{\alpha_{n} j} B_{1}^{x}\left(\alpha_{n}, \beta_{m}\right) \\
\Lambda_{1}\left(\alpha_{n}, \beta_{m}\right)=\frac{\lambda_{0}\left(\alpha_{n}^{2}+\beta_{m}^{2}\right)}{\beta_{m} j} B_{1}^{y}\left(\alpha_{n}, \beta_{m}\right)
\end{array} .\right.
$$

The variables $\alpha_{\mathrm{n}}$ and $\beta_{m}$ are the spatial pulsations of the double Fourier transform. The following relations define the spatial pulsations $\alpha_{\mathrm{n}}$ and $\beta_{m}$ :

$$
\alpha_{n}=\frac{2 n \pi}{L_{x}} ; \beta_{m}=\frac{2 m \pi}{L_{y}},
$$

where $L_{x}$, respectively $L_{y}$, is the distance upon which the Fourier transform is made along $O x$, respectively $O y$.

The particular cases where $\alpha_{n}=0$ or $\beta_{m}=0$ are treated separately, and give:

$$
\begin{aligned}
& \Lambda_{1}\left(\alpha_{n}, 0\right)=-\alpha_{n} j \lambda_{0} B_{1}^{x}\left(\alpha_{n}, 0\right), \\
& \Lambda_{1}\left(0, \beta_{m}\right)=-\beta_{m} j \lambda_{0} B_{1}^{y}\left(0, \beta_{m}\right) .
\end{aligned}
$$

Equations (9), (14), (16) and (17) are ill-posed problems for inverse techniques (Hensel 1991). The noise on the $\mathbf{b}_{1}$-field is increased by derivation, and the $\lambda_{1}$-field is even noisier than the $\mathbf{b}_{1}$-field. When $\alpha_{n} \neq 0$ and $\beta_{m} \neq 0$, the estimation of $\Lambda_{1}$ can, at first sight, be done with the first or the second equation of system (14). But the error upon the conductivity field depends on the values of $\alpha_{\mathrm{n}}$ and $\beta_{\mathrm{m}}$. Thus, in the first equation of system (14), large values of $\beta_{\mathrm{m}}$ will give larger errors upon $\lambda_{1}$. On the other hand, the second equation is more sensitive to $\alpha_{\mathrm{n}}$ values. So as to make the most accurate estimation of $\lambda_{1}$-field, the first or the second equation is chosen, according to the values of $\alpha_{\mathrm{n}}$ and $\beta_{\mathrm{m}}$. Instead of in 1D case, this latter remark, and Eqs. (16) and (17), make the estimation of $\mathbf{b}$-field with respect to two space directions absolutely necessary.

The inversion of the $\Lambda_{1}$ spectrum yields an estimation of the first order of the development of the conductivity field, $\lambda_{1}(x, y)$. This $\lambda_{1}$-field is a relative distribution of local thermophysical properties, and not an absolute measurement of the thermal conductivity.

\section{Experimental results}

The experimental devices are schematized on Fig. 1. The samples are made of perforated steel plates and epoxy resin. The surface of the samples is painted black so as to ensure a constant and close to 1 emissivity. The infrared pictures are obtained with an AVIO TVS 2000 infrared camera, which can record up to 512 images. Each image is composed of $100 \times 256$ pixels. Detailed informations about the size of the samples, the experimental precautions and the processing and inversion of the data are given in Varenne et al, 2000-a and Varenne et al, 2000-b.

Figure 2 illustrates an example of an instantaneous local temperature field $T$ of the surface of the sample in $1 \mathrm{D}$ and $2 \mathrm{D}$ case. The measurement noise hides the local temperature fluctuations, which will reappear thanks to the treatment of a great number of temperature fields. Figure 3 illustrates a row (along the $O x$ direction) of the $b$-field in 1D case and, in 2D case, $b_{1}^{x}$-field, whose width (along the $O y$ direction) is equal to the width of a unit cell. It shows that the estimation is more accurate for the points that are closer to the thermal excitation (smaller $x$ abscissas), where temperature gradients are larger. A similar estimation with the heating plane resistance on top of the image is made

to obtain the $b_{1}^{y}$ field. Figure 4 illustrates the power spectral density of $b_{1}^{x}$. The 
periodicity is very accurately identifiable, since the power peaks are sharply defined. The $b_{1}^{x}$-field corresponding to the observed surface can then be reconstructed by duplication of the average unit cell. In 1D case, the resistivity field(inverse of conductivity field) is obtained with Eq. (9) and inversion method. In 2D case, the image of the $b_{1}^{x}$-field in Fourier space is calculated. The same treatment is applied to $b_{1}^{y}$-field. The spectra $B_{1}^{x}$ and $B_{1}^{y}$ give an estimation of $\Lambda_{1}$ via system (14), Eq. (16) and Eq. (17), and therefore of $\lambda_{1}$, by the inverse Fourier transform of spectrum $\Lambda_{1}$. Figure 6 represents the 1D case estimation of the resistivity field and in 2D case, the first order of the estimated conductivity field $\lambda_{1}$. The medium's morphology is correctly estimated, although the angles of the square inclusions are smoothed by the estimation method. These smoothing effects are also partly due to the camera space filtering. But they are also reinforced by the treatment in itself, which attenuates the information with higher space frequencies.

The proposed method represents a fast and convenient experimental process to estimate the local thermal conductivity field of a 2D heterogeneous periodic medium. The morphology and the order of magnitude of the conductivities were accurately estimated.

\section{References}

Aziz A, 1988, In: Minkowycz W.J. et al., Handbook of numerical heat transfer, Wiley, N.Y. Beck J V, Arnold K J, 1977, Parameter estimation in engineering and science, Wiley, N.Y.

Carbonell R G, Whitaker S, 1984, In: Fundamentals of Transport Phenomena in Porous Media, Ed. J.Bear, N.Y.C., 121-198

Hensel E, 1991, Inverse theory and applications for engineers, Prentice Hall Advanced Reference Series - Engineering, Englewood Cliffs

Lepoutre F, Lefebvre J, Lhermitte T, Ainouch L, Delpech P, Forge P, Hirschi S, Joulaud J L, 1996, Rev. Gén. Thermique. 35 344-354

Marle C M, 1967, Rev. Inst. Fr. Petrole. 22 1471-1509

Nozad I, Carbonell R G, Whitaker S, 1985, Chem. Eng. Sci. 40 843-855

Quintard M, Whitaker S, 1993, Adv. in Heat Transfer. 23 369-464

Varenne M, Batsale J C, Gobbé C, 2000-a, Journal of Heat Transfer-ASME- 122, pp21-26

Varenne M, Batsale J C, Gobbé C, 2000-b, High Temperature High Pressure- 32, pp329-336
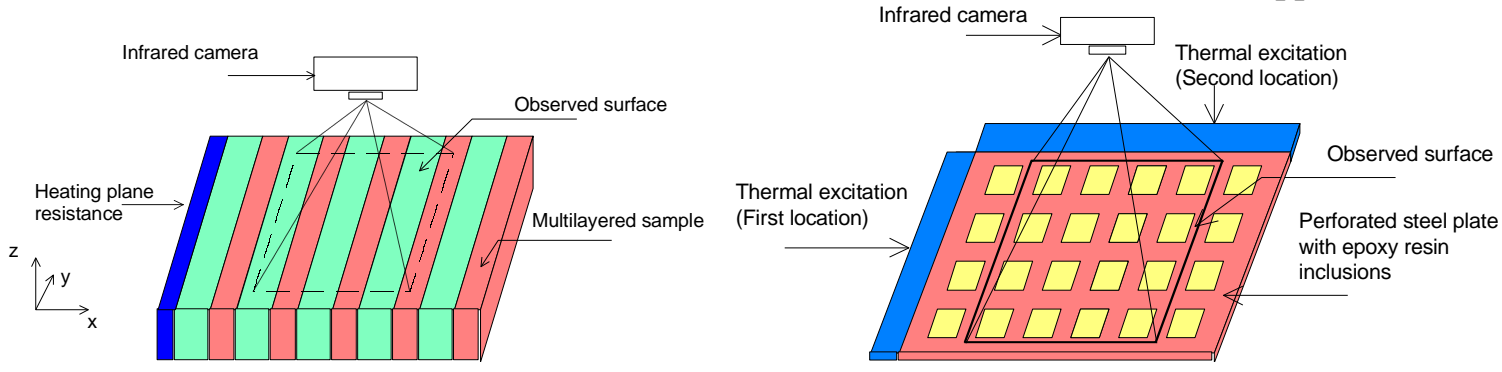

Figure 1 Schematic of the experimental calibration $1 D$ and 2D devices 

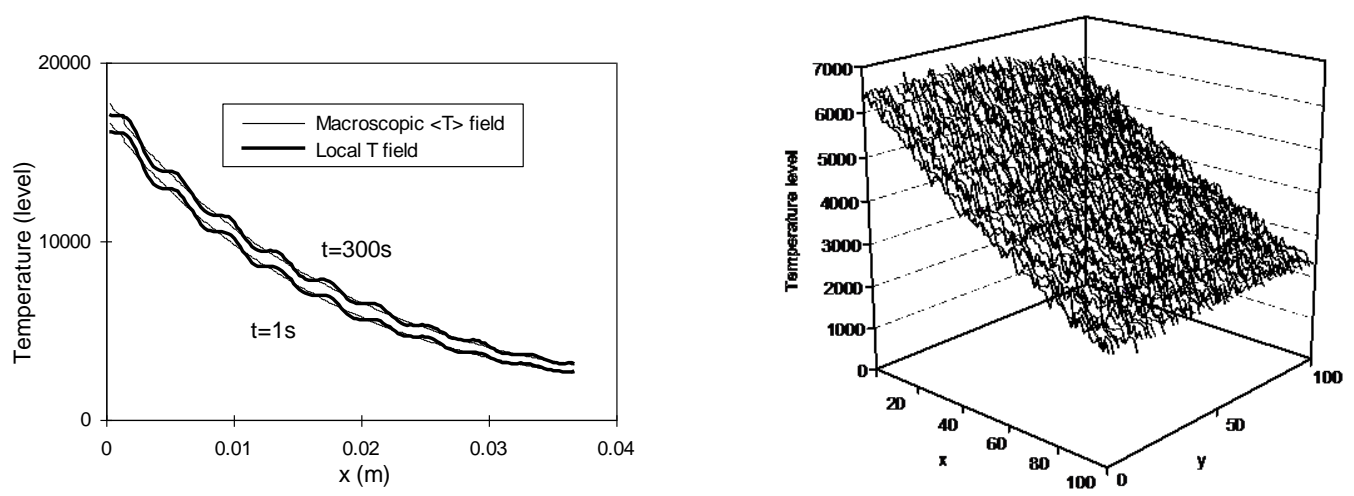

Figure 2 Instantaneous temperature fields in 1D and 2D cases
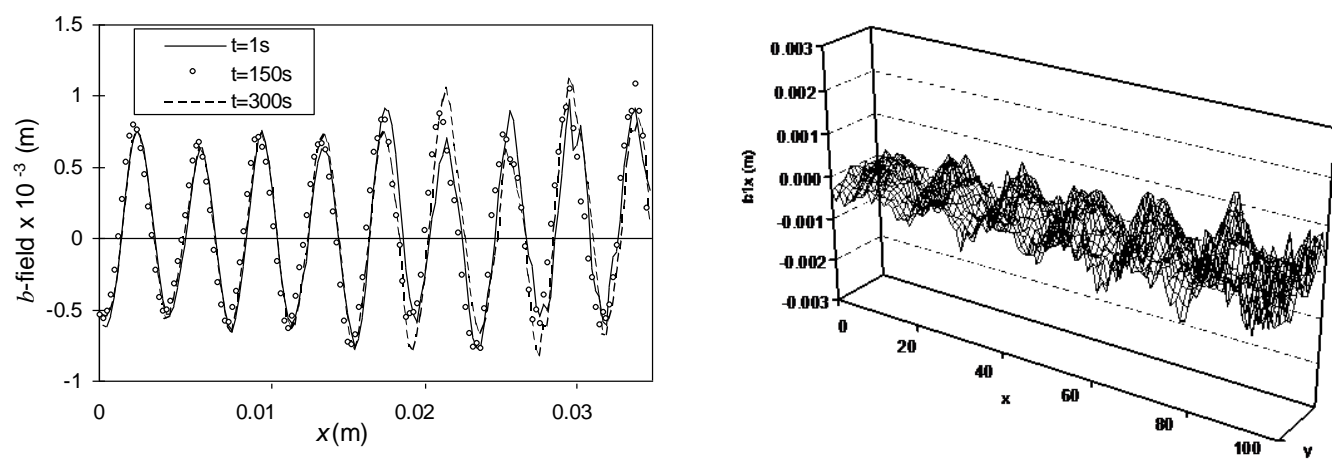

Figure 3. $b$ field in 1D case and $b_{1}^{x}$-field in 2D case

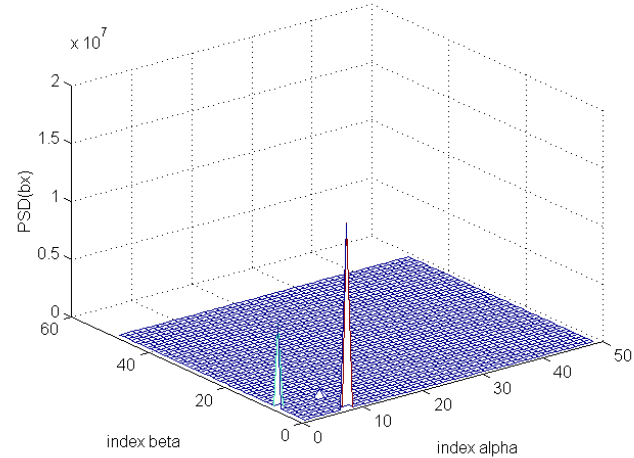

Figure 4. Power spectral density of $b_{1}^{x}$-field in 2D case
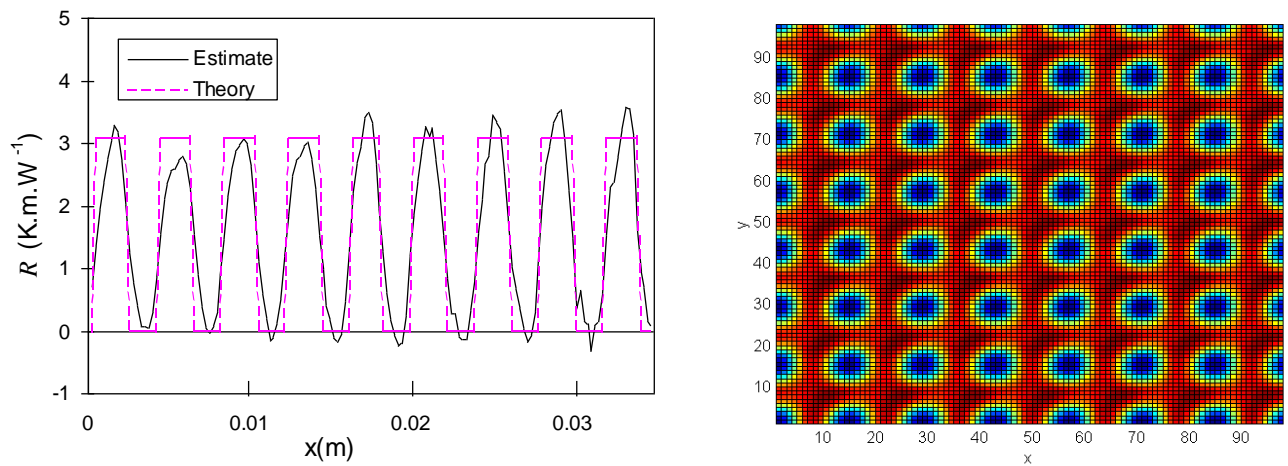

Figure 5 Thermal resistivity-field in 1D case and $\lambda_{1}$-field in 2D case 